\title{
Evaluation of Ofloxacin in the Treatment of Laparoscopically Documented Acute Pelvic Inflammatory Disease (Salpingitis)
}

\author{
J.F. Peipert, ${ }^{1 *}$ R.L. Sweet, ${ }^{2}$ C.K. Walker, ${ }^{3}$ J. Kahn, ${ }^{4}$ and \\ K. Rielly-Gauvin ${ }^{4}$ \\ ${ }^{1}$ Department of Obstetrics and Gynecology, Women $\&$ Infants Hospital of Rhode Island, \\ Providence, $R I$ \\ ${ }^{2}$ Department of Obstetrics and Gynecology and Reproductive Science, Magee-Women's Hospital, \\ Pittsburgh, $P A$ \\ ${ }^{3}$ Department of Reproductive Infectious Diseases and Immunology, University of California at \\ Irvine School of Medicine, Orange, CA \\ ${ }^{4}$ Ortho-McNeil Pharmaceutical, Raritan, $N J$
}

\begin{abstract}
Objective: To evaluate the safety and efficacy of intravenous and oral ofloxacin monotherapy in the treatment of laparoscopically documented acute pelvic inflammatory disease (PID).

Methods: This study was conducted as an open-label, phase-III, uncontrolled, multicenter study. Patients identified with laparoscopic findings of salpingitis were treated with $400 \mathrm{mg}$ of intravenous ofloxacin every 12 hours followed by $400 \mathrm{mg}$ of oral ofloxacin every 12 hours for 10 to 14 days. Patients were evaluated five times for clinical and microbial efficacy. Since laparoscopy was performed only at admission, pathogens identified laparoscopically were presumed eradicated if they were present on the laparoscopic culture and the patient was clinically cured or improved at final evaluation.

Results: Of the 70 patients evaluable for safety (intent-to-treat population), the mean age was 25.6 years. Sixty-one of 70 patients $(87 \%)$ were cured, one improved, one did not improve, and seven were unevaluable because they discontinued study participation. Fifty-one were evaluable for clinical efficacy: $50(98 \%)$ were cured and one did not improve. Sixteen were evaluable for expanded microbiological efficacy: three had documented Neisseria gonorrhoeae; 12, Chlamydia trachomatis, and one, a mixed infection of both organisms. All cervical, laparoscopic, and endometrial cultured pathogens, including $N$. gonorrhoeae and $C$. trachomatis, were eradicated or presumed eradicated at the posttherapy visit. No serious or unexpected adverse events occurred.

Conclusions: Ofloxacin monotherapy was effective and well tolerated in the treatment of laparoscopically proven PID in a geographically diverse population. Future studies are necessary to evaluate long-term outcomes and sequelae of PID treatment with single agent therapy. Infect. Dis. Obstet. Gynecol. 7:138-144, 1999. @ 1999 Wiley-Liss, Inc.
\end{abstract}

KEY WORDS

fluoroquinolone; sexually transmitted disease; Neisseria gonorrhoeae; Chlamydia trachomatis;

adnexitis; salpingitis

O ver the past two decades, significant changes have occurred in the accepted etiology, diagnosis, and treatment of acute pelvic inflammatory disease (PID). Neisseria gonorrhoeae, once viewed as the primary etiologic agent, is now one of several microorganisms associated with PID. $N$. gonor-

*Correspondence to: Dr. Jeffrey F. Peipert, Department of Obstetrics and Gynecology, Women \& Infants Hospital of Rhode Island, 101 Dudley Street, Providence, RI 02905-2401. E-mail: jpeipert@wihri.org 
rhoeae, Chlamydia trachomatis, gram-negative enteric bacteria, and pelvic anaerobes all have been isolated from the upper genital tract of women diagnosed with acute PID. ${ }^{1-4}$

Additionally, PID is often found to be a polymicrobial infection ${ }^{5}$ with varying and complex presentations of signs and symptoms. Due to the probability of disease progression and the development of serious long-term sequelae, immediate treatment of suspected PID with a broad-spectrum antibiotic is recommended. To provide antimicrobial activity against possible multiple pathogenic organisms, the Centers for Disease Control and Prevention (CDC) guidelines for the treatment of PID recommend combination therapy regimens. ${ }^{6}$ For oral treatment, these guidelines include the use of the fluoroquinolone ofloxacin in combination with metronidazole.

Ofloxacin has demonstrated in vitro activity against $N$. gonorrhoeae (including penicillinresistant strains), $C$. trachomatis, gram-positive and gram-negative aerobic bacteria, and some anaerobic bacteria. Additionally, it has been shown that the pharmacokinetic properties of ofloxacin include high levels of drug distribution in gynecologic tissues ${ }^{7}$ and $100 \%$ oral bioavailability, which allows direct-dose conversion between parenteral and oral therapy. The ability to eradicate a broad spectrum of microorganisms and the ability to convert directly from intravenous (IV) to oral administration enhance the clinical usefulness of ofloxacin in the treatment of PID.

Soper et al. previously reported a clinical success rate (cure plus improvement) of $100 \%$ for ofloxacin monotherapy in the treatment of patients who had $N$. gonorrhoeae as the dominant cause of PID. ${ }^{8}$ The objective of our multicenter, open-label study was to evaluate the safety and efficacy of IV and oral ofloxacin monotherapy in the treatment of laparoscopically documented acute salpingitis in diverse patient populations.

\section{SUBJECTS AND METHODS}

Women were eligible for participation if they had clinical signs and symptoms consistent with a diagnosis of acute salpingitis (i.e., lower abdominal direct tenderness, adnexal tenderness, and pain on motion of cervix or uterus) plus one of the following: oral temperature $>38.3^{\circ} \mathrm{C}$, mucopurulent cervical discharge, vaginal leukorrhea, elevated eryth- rocyte sedimentation rate, or elevated C-reactive protein. Exclusion criteria included women who were lactating, currently pregnant, less than 18 years of age, postmenopausal, using an intrauterine device, grossly underweight $(40 \mathrm{~kg})$, inappropriate candidates for quinolone therapy, not suitable candidates for diagnostic laparoscopy, those who had acquired immunodeficiency syndrome (AIDS), tubo-ovarian abscess, active seizures, or major psychiatric disorders, and those who had undergone surgery for tubal ligation or hysterectomy. Patients were also excluded from the study if they were critically ill with conditions requiring immediate surgery, had participated previously in this protocol, required concomitant systemic antimicrobial therapy, or were exposed to any investigational drug within the previous 30 days or to more than one dose of any nontopical antimicrobial therapy within the previous 48 hours. A geographically diverse study population was established by including 71 patients from California, Florida, Illinois, Louisiana, Pennsylvania, New York, and Rhode Island. Prior to beginning the study protocol, Institutional Review Board approval was obtained at all seven participating sites in the U.S. Before study admittance, eligible patients signed an informed consent form for participation.

To be evaluable, all women were required to have documented laparoscopic evidence of salpingitis (described below). Immediately following laparoscopic confirmation, eligible patients were treated with $400 \mathrm{mg}$ of IV ofloxacin every 12 hours. When an investigator determined that the patient was able to tolerate oral medications, $400 \mathrm{mg}$ of oral ofloxacin was administered every 12 hours for a total treatment duration (IV and oral medication) of 10 to 14 days.

Patients were evaluated at admission, 48 hours after initiation of ofloxacin therapy, before conversion to oral therapy, 3 to 15 days posttherapy (or at early withdrawal), and 3 to 5 weeks after completion of therapy (long-term follow-up visit). Assessment of clinical signs and symptoms at admission, at 48 hours after the initiation of therapy, and at both the posttherapy and long-term follow-up visit included evaluation of abdominal pain, dysuria, nausea/vomiting, fever, vaginal leukorrhea, and mucopurulent cervical discharge. Physical examinations and vital signs were recorded at admission, 48 hours after initiation of therapy, before conver- 
sion to oral therapy, and at posttherapy or early withdrawal. Assessment of PID included the establishment of a modified severity score based on the method of McCormack et al. ${ }^{9}$ This PID severity score was calculated by graded assessments (absent, minimal, moderate, and severe) of lower abdominal direct tenderness, adnexal tenderness, pain on motion of the cervix or uterus, and rebound tenderness. Samples for serum chemistry, hematology, and urinalysis were collected at admission, before conversion to oral therapy, and at the posttherapy visit. Sexual history was reviewed at the posttherapy and long-term visits.

In the current trial, a single laparoscopic examination at admission was used for the diagnosis of acute salpingitis. The admission (pretreatment) laparoscopic evaluation determined the presence (or absence) of Fitz-Hugh-Curtis syndrome (i.e., presence of acute right upper quadrant pain and tenderness in association with acute salpingitis) ${ }^{1}$ and identified criteria specified by Westrom (Student thesis, Lund, Sweden; 1976) as follows (1) redness of fallopian tube surface, (2) edema of tubal wall, and (3) purulent or seropurulent exudate covering tubal surface or leaking from fimbriae. The severity of each Westrom criterion was rated as none, mild, moderate, or severe by the investigator. Patients were considered to have laparoscopically documented acute salpingitis if (1) all three Westrom criteria were present or (2) at least one Westrom criterion was present combined with a positive admission culture of $N$. gonorrhoeae and/ or $C$. trachomatis from either the laparoscopically obtained or endometrial biopsy specimens. This definition was chosen to maximize the sensitivity (minimizing the occurrence of false negatives) of laparoscopic evaluations. ${ }^{10}$

Clinical response was determined by the investigator 3 to 15 days following completion of ofloxacin therapy. Clinical response was assessed as either cured, improved, failed (no improvement), or unable to evaluate.

To determine microbiological efficacy, samples for culture from the cervix and endometrium were obtained at admission and during laparoscopy from a fallopian tube puncture or fimbrial exudate. For this study, pathogens were defined as isolates of $N$. gonorrhoeae and $C$. trachomatis. In addition, all isolated aerobic organisms, with the exception of $C$. trachomatis, were tested according to the National
Committee for Clinical Laboratory Standards (NCCLS) disk susceptibility protocol, ${ }^{11}$ and all organisms (aerobes and anaerobes) were evaluated per NCCLS minimum inhibitory concentration (MIC) determination protocols. ${ }^{12,13}$

All culture samples also were analyzed for $C$. trachomatis by a polymerase chain reaction (PCR) assay. Repeat culture samples for $G$. trachomatis only were obtained from the cervix and, if positive at admission, from the endometrium during the posttherapy and long-term visits.

In vivo microbiological response to ofloxacin therapy was determined for $N$. gonorrhoeae and $C$. trachomatis. Positive admission endometrial and cervical culture results were compared to culture results obtained from same-site samples collected during the posttherapy visit. Microbiological response for endometrial and cervical culture results was assessed as eradicated, persisted, new infection (i.e., new pathogen present), or unable to evaluate. Since laparoscopy was performed only at admission, only presumed microbiological response based on clinical response could be determined for laparoscopically isolated pathogens. If a pathogen was present at laparoscopy and the patient was clinically cured or improved at the posttherapy visit, the organism was presumed to be eradicated. Similarly, if the patient was assessed as a clinical failure posttherapy, any laparoscopically obtained pathogen was presumed to have persisted.

Additionally, all patients who received at least one dose of ofloxacin (intent-to-treat population) were evaluated for safety throughout the entire study (up to 5 weeks posttherapy). All adverse events were documented by the investigator and assessed as mild, moderate, or marked. The relationship of adverse event to study drug also was assessed by the investigator as none, unlikely, possible, probable/likely, or certain.

Efficacy parameters were summarized and tabulated without any inferential statistical analysis. Vital signs (oral temperature, respiratory rate, pulse rate, and systolic and diastolic blood pressure) were compared using the Student paired $t$ test (twotailed) to test the null hypothesis that the means of the vital sign measurements at admission and posttherapy were equal. The changes in laboratory test results (hematology, blood chemistry, and urinalysis tests) were analyzed using a modified McNemar 
statistical test ${ }^{14}$ based on the chi-square distribution with one degree of freedom and a correction of continuity. The test was based on changes in investigator-supplied normal ranges. All statistical inferences were based on type-I error rate of 0.05 . All statistical appendices and tabulations were generated using the Statistical Analysis System (SAS). ${ }^{15}$

\section{RESULTS}

A total of 70 patients received at least one dose of ofloxacin and were evaluated for safety. Sixty-two patients ( $89 \%$ ) had the diagnosis of acute PID confirmed at laparoscopy. Nineteen of the 70 patients were excluded from the analysis of clinical efficacy: 10 were excluded due to protocol violations, eight patients had a negative laparoscopic evaluation, and one patient did not have a final assessment. Fifty-one patients were therefore evaluable for clinical efficacy. Seven patients were considered microbiologically evaluable based on positive cultures for $N$. gonorrhoeae or $G$. trachomatis from laparoscopic and/or endometrial specimens at admission. Sixteen patients were evaluable for expanded microbiological efficacy based on cultures from laparoscopic, endometrial, or cervical specimens.

Of the clinically evaluable study population ( $N$ $=51$ ), $24 \%$ were white and $63 \%$ were black, and the mean age was 25.1 years. On admission, 50 patients (98\%) reported abdominal pain, 27 (53\%) had nausea/vomiting, and 19 (37\%) had dysuria. Mucopurulent cervical discharge was noted in $42(82 \%)$, and $38(75 \%)$ had leukorrhea. Three patients $(6 \%)$ had a temperature above $38.5^{\circ} \mathrm{C}$ and 11 patients (22\%) had an abnormal white blood cell count $(\geq 15,000$ or $<5,000)$. Eighteen patients $(35 \%)$ were noted to have Fitz-Hugh-Curtis syndrome on laparoscopic evaluation. Microbiological results were as follows: 16 patients ( $31 \%$ ) had positive cultures for $N$. gonorrhoeae and/or $C$. trachomatis, 20 (39\%) had positive cultures for other organisms, and 15 (29\%) had negative culture results. Six types of anaerobic bacteria were isolated from the endometrium a total of 13 times (Table 1).

The mean number of days of IV ofloxacin therapy was 2.3 prior to conversion to oral therapy. The mean number of treatment days for both regimens (IV and oral) was 13.6 (range: 9 to 16.5 days).
TABLE I. Anaerobic bacteria isolated from the endometrium at admission

\begin{tabular}{lc}
\hline Organism & No. of times isolated \\
\hline Bacteroides spp. & 4 \\
Bacteroides fragilis & 3 \\
Clostridium perfringens & 1 \\
Lactobacillus spp. & 3 \\
Peptostreptococcus spp. & 1 \\
Peptostreptococcus anaerobis & 1 \\
\hline
\end{tabular}

At the posttherapy visit 50 of the 51 clinically evaluable patients with a history of PID (98\%) were assessed as clinically cured. One patient had negative culture and PCR determinations at admission and was considered a treatment failure at the 3 - to 15-day posttreatment evaluation. In accordance with a protocol provision, this patient remained on ofloxacin therapy with the addition of metronidazole and was subsequently cured of her current episode of PID.

Patients who were clinically evaluable at 3-15 days posttherapy had significant improvement in PID signs and symptoms. At admission, abdominal pain, nausea/vomiting, and dysuria, were present in 98\%, 53\%, and 37\%, respectively. Posttherapy, $4 \%$ (2/51) had abdominal pain, $8 \%$ (4/51) had nausea/ vomiting, and $4 \%(2 / 51)$ had dysuria. Those symptoms that were present postherapy were mild in severity, with the exception of one case of severe dysuria. Scores of PID also showed improvement at posttherapy. At admission, 31 patients (61\%) had a PID score of at least 7 , and 20 patients $(39 \%)$ had a score between 1 and 6 . At the posttherapy visit, 47 patients $(92 \%)$ who were clinically evaluable had a PID score of 0 , and $4(8 \%)$ had a residual PID score of 1 to 3 , indicating minimal persistence of a single symptom. Of these 4 , the one patient with a residual McCormack PID score of 3 was considered a clinical failure.

In order to assess any relapse or reinfection, the severity of signs and symptoms present at admission was compared to the severity of signs and symptoms persisting 3 to 5 weeks posttherapy. The efficacy of ofloxacin was confirmed by the transition of signs and symptoms at the long-term visit, where there was no clinical evidence of relapse or reinfection. Among the clinically evaluable population, $90 \%$ or more had no signs or symptoms of pain, dysuria, mucopurulent cervical discharge, 
vaginal leukorrhea, or nausea and/or vomiting. The only reported signs and symptoms present at longterm follow up that were not present on admission were two instances of mild-to-moderate dysuria and one report of mild nausea and vomiting.

Among 51 clinically evaluable patients, three had documented infection due to $N$. gonorrhoeae, 12 had documented infection due to $G$. trachomatis, and one had a mixed infection due to both organisms. As expected, the initial site of these pathogens varied. C. trachomatis alone was isolated in 12 patients at the following sites: cervix (8); cervix and endometrium (1); cervix and at laparoscopy (1); and cervix, endometrium, and at laparoscopy (2). $N$. gonorrhoeae alone was isolated in three patients at the following initial sites: cervix (1); endometrium (1); and cervix, endometrium, and at laparoscopy (1). Additionally, one patient had a cervical culture positive for both $C$. trachomatis and $N$. gonorrhoeae coupled with an endometrial culture positive only for $N$. gonorrhoeae. In 16 microbiologically evaluable patients with organisms from laparoscopic, endometrial, and/or cervical specimens isolated at admission, all $N$. gonorrhoeae and $C$. trachomatis organisms were eradicated or presumed eradicated at the 3- to 15-day posttherapy evaluation. Furthermore, six anaerobic organisms were isolated from 13 patients (see Table 1), primarily from the endometrial sites, at admission. At the posttherapy visit, all patients with anaerobic isolates identified at admission were considered clinically cured.

In vitro ofloxacin susceptibility testing was performed on all organisms isolated at admission, with the exception of $C$. trachomatis. These data include all patients who were not clinically evaluable. $N$. gonorrhoeae was isolated nine times: seven isolates were susceptible to ofloxacin, and two had no data reported. Twenty-two other organisms were isolated 55 times: 25 isolates were susceptible to ofloxacin, three had intermediate susceptibility, three were resistant, and 24 had no susceptibility results (see Table 2).

A long-term follow-up visit was not required for completion of the study; however, efforts were made 3 to 5 weeks posttherapy to obtain patient culture specimens from cervical and endometrial sites. Sixteen patients who had pathogens isolated at admission showed negative cervical and/or endometrial cultures for $G$. trachomatis and $N$. gonorrhoeae at the long-term follow-up visit. $N$. gonor-
TABLE 2. In vitro ofloxacin susceptability at admission ${ }^{\mathrm{a}}$

\begin{tabular}{|c|c|c|c|}
\hline Organism & Susceptible & $\begin{array}{l}\text { Intermediate } \\
\text { susceptibility }\end{array}$ & Resistant \\
\hline Bacteroides & 1 & & \\
\hline Bacteroides fragilis & & 3 & \\
\hline \multicolumn{4}{|l|}{ Clostridium perfringens } \\
\hline \multicolumn{4}{|l|}{ Corynebacterium } \\
\hline Escherichia coli & 3 & & \\
\hline Gardnerella vaginalis & 5 & & \\
\hline Klebsiella pneumoniae & 1 & & \\
\hline \multicolumn{4}{|l|}{ Lactobacillus } \\
\hline \multicolumn{4}{|l|}{ Neisseria mucosa } \\
\hline \multicolumn{4}{|l|}{ Peptostreptococcus } \\
\hline $\begin{array}{l}\text { Peptostreptococcus } \\
\text { anaerobis }\end{array}$ & 1 & & \\
\hline Pseudomonas & 1 & & \\
\hline $\begin{array}{l}\text { Coagulase-negative } \\
\text { staphylococci }\end{array}$ & 3 & & \\
\hline Staphylococcus aureus & 1 & & \\
\hline $\begin{array}{l}\text { Staphylococcus } \\
\text { epidermidis }\end{array}$ & 2 & & I \\
\hline Staphylococcus hominis & $\mathrm{I}$ & & \\
\hline \multicolumn{4}{|l|}{ Streptococcus } \\
\hline Streptococcus agalactiae & 2 & & \\
\hline Streptococcus faecalis & 3 & & 2 \\
\hline \multicolumn{4}{|l|}{ Streptococcus group F } \\
\hline \multicolumn{4}{|l|}{ Torulopsis glabrata } \\
\hline $\begin{array}{l}\text { Unknown gram- } \\
\text { positive organism }\end{array}$ & I & & \\
\hline Total & 25 & 3 & 3 \\
\hline
\end{tabular}

an vitro susceptibility testing was performed on all organisms isolated at admission except $C$. trachomatis. $N$. gonorrhoeae was isolated nine times: seven isolates were susceptible to ofloxacin, and two had no data reported.

rhoeae was not present among patients at the longterm follow-up evaluation. One patient with a positive cervical culture only for $N$. gonorrhoeae at admission was found to have a positive cervical culture only for $C$. trachomatis at the long-term follow-up evaluation. At the 3- to 15-day posttherapy visit, however, this patient had a negative cervical culture for both organisms and was assessed as microbiologically cured. This patient had resumed unprotected sexual activity after the 3- to 15-day posttherapy visit; thus, the long-term follow-up positive cervical culture for $C$. trachomatis may have been due to a new sexually transmitted infection.

Of the 70 patients evaluable for safety, 17 (24\%) reported at least one adverse event related to the study drug. No deaths or serious adverse events occurred during the study. The most common side effect was a reported fungal infection experienced by 11 patients $(15.7 \%)$. Seven patients $(10 \%)$ experienced nausea. Two patients $(3 \%)$ experienced 
a nervous system complaint, and one (1.4\%) experienced a dermatologic complaint.

\section{DISCUSSION}

Ofloxacin monotherapy is reported to be as effective as the traditional therapy of cefoxitin and doxycycline for the treatment of PID. Results from two comparative studies of women with PID showed a clinical success rate (cured plus improved patients) for ofloxacin monotherapy of $95 \%$ in a total of 165 patients, compared with clinical success rates of $93 \%$ to $97 \%$ reported among 156 patients treated with cefoxitin and doxycycline. ${ }^{16,17}$ Consistent with these findings, a noncomparative double laparoscopic study of 36 patients treated with ofloxacin showed a $100 \%$ clinical success rate. ${ }^{8}$

This clinical evaluation of 70 patients with diverse demographic and geographic characteristics supports the previously reported findings regarding the use of ofloxacin as monotherapy for the treatment of acute PID. Although this was an uncontrolled study, of the 51 clinically evaluable patients, $50(98 \%)$ were clinically cured after ofloxacin treatment. The one patient assessed as a clinical failure had a prior history of Chlamydia-associated PID; however, her pathogen culture and PCR results were negative at admission. This patient was clinically cured with continued ofloxacin therapy and the addition of metronidazole in accordance with the protocol.

Microbiological response to ofloxacin treatment for the 16 patients with cultures positive for $N$. gonorrhoeae and/or $C$. trachomatis was $100 \%$ eradicated (confirmed or presumed) at 3 to 15 days posttherapy. These results are comparable to other well-controlled studies of ofloxacin treatment in women with PID, which have reported $100 \%$ eradication of $N$. gonorrhoeae and 86 to $100 \%$ eradication of $G$. trachomatis. $8,16,17$ Sites of pathogens at admission were consistent with other studies in that the majority of pathogens ( 9 of 16) were isolated from the cervix. However, the percentage of patients (31\%) with both laparoscopically documented salpingitis and culture findings positive for these organisms was significantly lower than previously reported. This difference may be due to the geographically diverse patient population or a possible high prevalence of women with PID due to reinfection by undetectable levels of $C$. trachomatis. Soper et al., who reported on a more geographically homogeneous population, found isolation of $N$. gonorrhoeae and/or C. trachomatis in 31 (86\%) of 36 patients with laparoscopically documented salpingitis. $^{8}$

In this study, seven isolates of the pathogen $N$. gonorrhoeae and 25 isolates of other aerobic and anaerobic organisms were susceptible to ofloxacin (see Table 2). Additionally, 13 anaerobic isolates were identified (see Table 1). One isolate of Peptostreptococcus anaerobius and one Bacteroides isolate were susceptible to ofloxacin. Three Bacteroides isolates had unknown sensitivities (not tested). The three Bacteroides fragilis isolates had an intermediate susceptibility to ofloxacin. Therefore, of the five anaerobic isolates tested, all were susceptible to ofloxacin; no anaerobes were found to be resistant. Prevotella was not isolated from any patient in this study.

The high clinical cure rate of ofloxacin in this study, combined with the in vitro studies, suggests that ofloxacin is effective in curing PID caused by multiple pathogens, including possible anaerobic pathogens. This successful outcome may be due to the high concentration of ofloxacin in the gynecologic tissues during treatment. ${ }^{7}$ However, this study did not have a long-term follow-up period to determine whether treatment prevented long-term sequelae. If the anaerobic organisms are not completely eradicated, they may persist and increase the risk of relapses. As has been shown in the study by Sweet et al., some patients treated for acute salpingitis have persistent chlamydial infection despite apparent clinical cure, which might cause chronic fallopian tube infection. ${ }^{18} \mathrm{~A}$ separate concern raised by some authorities is that a 14-day course of quinolone therapy may select for quinolone-resistant Pseudomonas species.

With respect to the safety evaluation, no deaths or serious adverse events occurred. The type and incidence of adverse events that were noted did not differ significantly from what has been previously associated with ofloxacin treatment.

In conclusion, this study supports an accumulating body of evidence that ofloxacin monotherapy is a safe and effective treatment for acute salpingitis. With the addition of this study to the medical literature, clinical success rates of $95 \%$ to $100 \%$ have been achieved with ofloxacin monotherapy for the treatment of PID in more than 250 clinically evaluable patients. Based on these findings, a 10 - to 
14-day treatment of IV ofloxacin with conversion after approximately 2 days to oral therapy is effective in the treatment of laparoscopically documented acute salpingitis. Additionally, ofloxacin, as either an IV or oral formulation, is generally safe and well tolerated in women being treated for acute PID. Additional studies, however, are needed to determine if persistent pathogens will present a clinical problem, and if long-term outcomes are similar in patients treated with ofloxacin or other standard therapies.

\section{ACKNOWLEDGMENTS}

Joseph Bellina, MD; Andrew Kaunitz, MD; Charles Livengood, MD; Subir Roy, MD; and Miklos Toth, MD, were participating investigators in the study.

\section{REFERENCES}

1. Sweet RL, Gibbs RS. Pelvic inflammatory disease. In: Infectious Diseases of the Female Genital Tract. 3rd ed. Baltimore, MD: Williams \& Wilkins, 1995. p 378-428.

2. Eschenbach DA, Buchanan TM, Pollock HM, et al. Polymicrobial etiology of acute pelvic inflammatory disease. N Engl J Med 1975;293:166-171.

3. Toomey KE, Rafferty MP, Stamm WE. Unrecognized high prevalence of Chlamydia trachomatis cervical infection in an isolated Alaskan Eskimo population. JAMA 1987;258:53-56.

4. Schachter J, Stoner E, Moncada J. Screening for chlamydial infections in women attending family planning clinics. Evaluation of presumptive indicators for therapy. West J Med 1983;138:375-379.

5. Jossens MOR, Schachter J, Sweet RL. Risk factors associated with pelvic inflammatory disease of differing antimicrobial etiologies. Obstet Gynecol 1994;83:989997.

6. Centers for Disease Control and Prevention: Pelvic inflammatory disease. In: 1998 Guidelines for the Treatment of Sexually Transmitted Diseases. MMWR 1998;47(RR-1):79-85.

7. Monk JP, Campoli-Richards DM. Ofloxacin: review of its antibacterial activity, pharmacokinetic properties and therapeutic use. Drugs 1987;33:346-391.
8. Soper DE, Brockwell NJ, Dalton HP. Microbial etiology of urban emergency department with acute salpingitis: treatment with ofloxacin. Am J Obstet Gynecol 1992; 167:653-660.

9. McCormack WM, Nowroozi K, Alpert S, et al. Acute pelvic inflammatory disease: characteristics of patients with gonococcal and nongonococcal infection and evaluation of their response to treatment with aqueous procaine penicillin $G$ and spectinomycin hydrochloride. Sex Transm Dis 1977;4:125-131.

10. Peipert JF, Boardman LA, Sung CJ. Performance of clinical and laparoscopic criteria for the diagnosis of upper genital tract infection. Infect Dis Obstet 1998;5: 291-296.

11. Performance Standards for Antimicrobial Disk Susceptibility Tests. 5th ed. Approved Standard 1993. National Committee for Clinical Laboratory Standards 13(24) (order code M2-A5).

12. Methods for Dilution Antimicrobial Susceptibility Test for Bacteria That Grow Aerobically. 3rd ed. Approved Standard 1993. National Committee for Clinical Laboratory Standards, 13(25) (order code M7-A3).

13. Alternative Methods for Antimicrobial Susceptibility Testing of Anaerobic Bacteria. 3rd ed. Approved Standard 1993. National Committee for Clinical Laboratory Standards 13(26) (order code M11-A3).

14. Fleiss J (ed). Statistical Methods for Rates and Proportions. 2nd ed. New York: John Wiley \& Sons Inc, 1981.

15. SAS (Statistical Analysis System): User's Guide, Statistics. 5th ed. Cary, NC: SAS Institute, 1985.

16. Martens MG, Gordon S, Yarborough DR, Faro S, Binder D, Berkeley A. Multicenter randomized trial of ofloxacin versus cefoxitin and doxycycline in outpatient treatment of pelvic inflammatory disease. South Med 1993; 86:604-610.

17. Wendel GD, Cox SM, Bawdon RE, Theriot SK, Heard MC, Nobles BJ. A randomized trial of ofloxacin versus cefoxitin and doxycycline in the outpatient treatment of acute salpingitis. Am J Obstet Gynecol 1991;164:1390 1396.

18. Sweet RL, Schachter J, Robbie MO. Failure of betalactam antibiotics to eradicate Chlamydia trachomatis in the endometrium despite apparent clinical cure of acute salpingitis. JAMA 1983;250:2641-2645. 


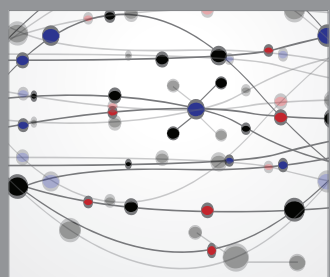

The Scientific World Journal
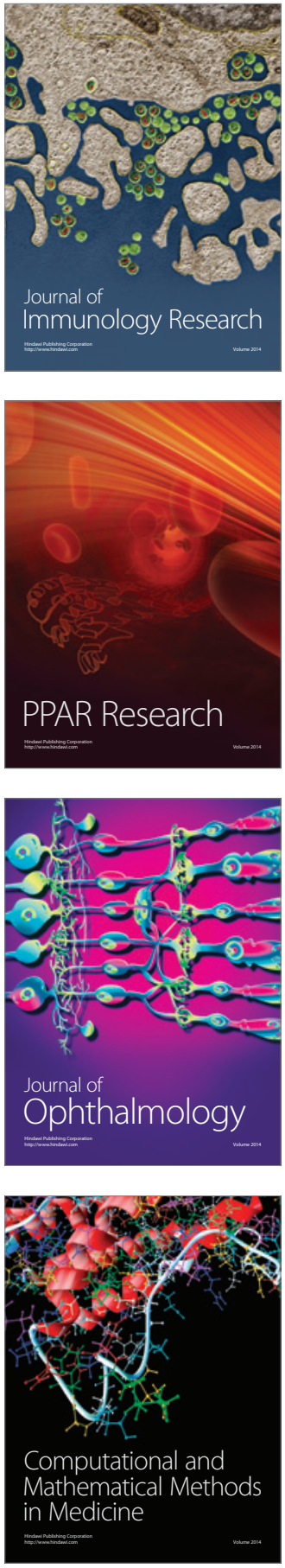

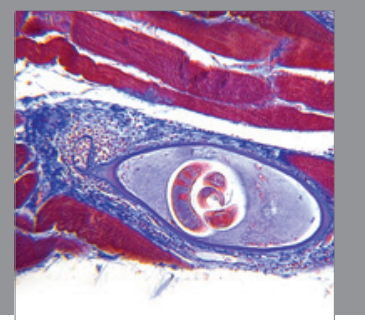

Gastroenterology

Research and Practice
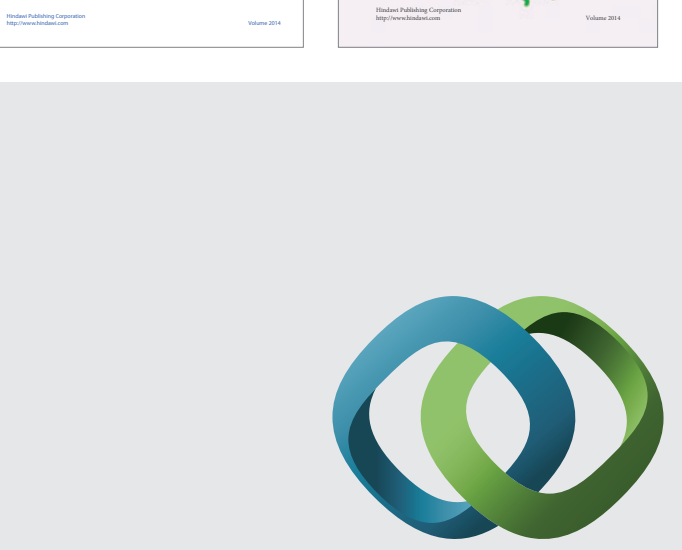

\section{Hindawi}

Submit your manuscripts at

http://www.hindawi.com
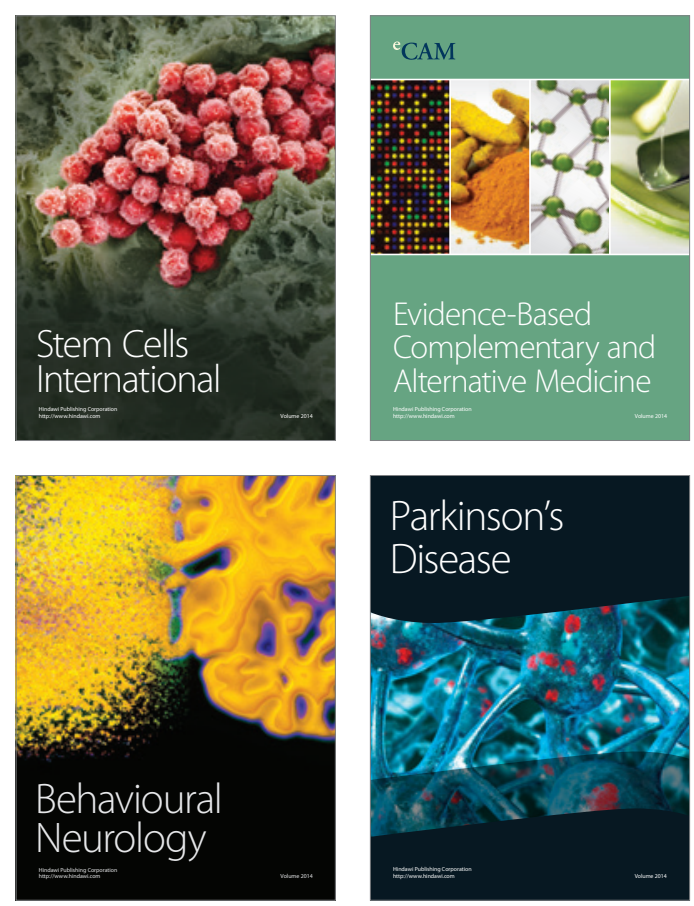

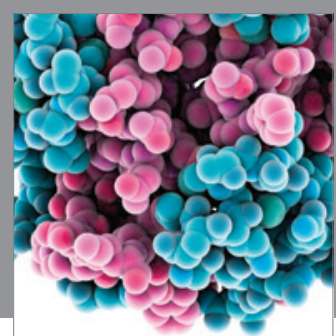

Journal of
Diabetes Research

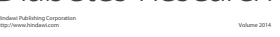

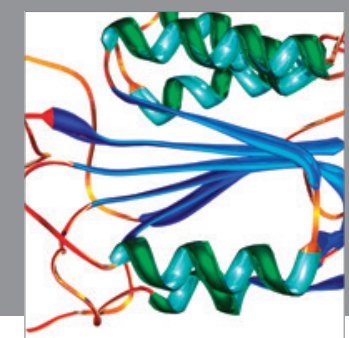

Disease Markers
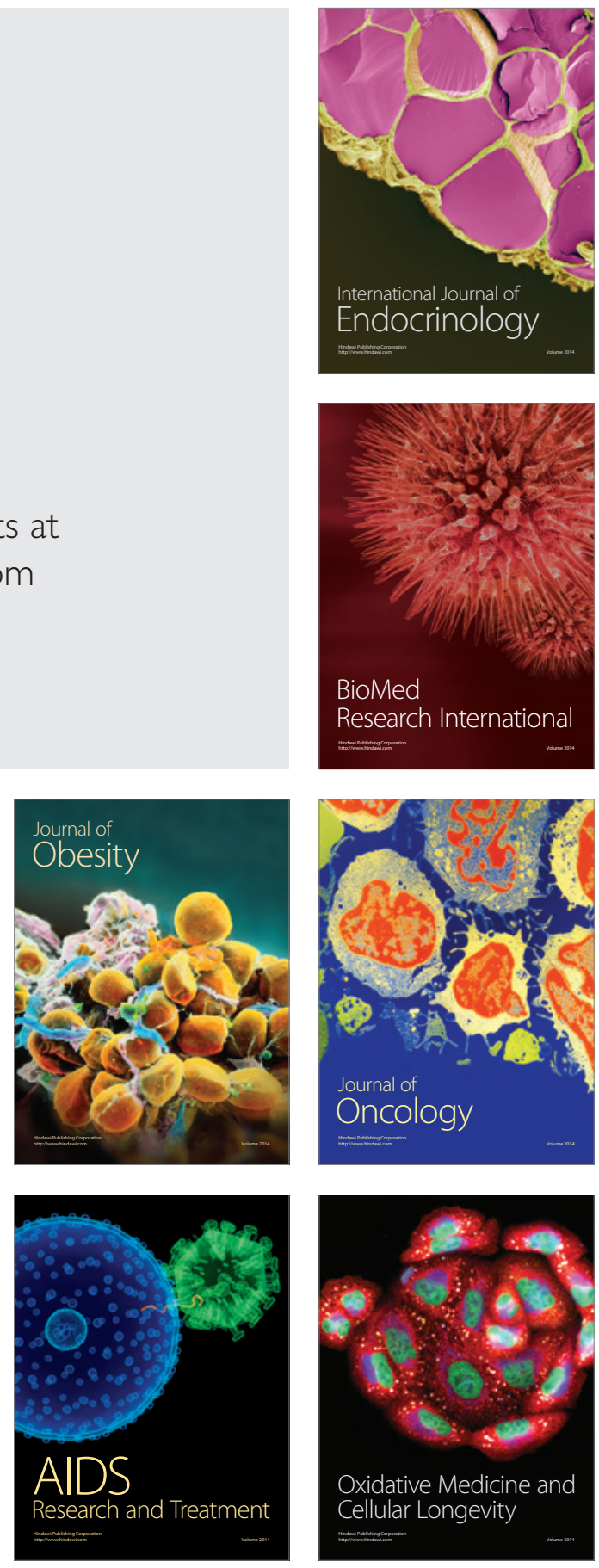